\title{
New roles for perforins and proteases in apicomplexan egress
}

Marijo S. Roiko and Vern B. Carruthers*

Department of Microbiology and Immunology, University of Michigan, Ann Arbor, MI 48109, USA.

\section{Summary}

Egress is a pivotal step in the life cycle of intracellular pathogens initiating the transition from an expiring host cell to a fresh target cell. While much attention has been focused on understanding cell invasion by intracellular pathogens, recent work is providing a new appreciation of mechanisms and therapeutic potential of microbial egress. This review highlights recent insight into cell egress by apicomplexan parasites and emerging contributions of membranolytic and proteolytic secretory products, along with host proteases. New findings suggest that Toxoplasma gondii secretes a poreforming protein, TgPLP1, during egress that facilitates parasite escape from the cell by perforating the parasitophorous membrane. Also, in a cascade of proteolytic events, Plasmodium falciparum latestage schizonts activate and secrete a subtilisin, PfSUB1, which processes enigmatic putative proteases called serine-repeat antigens that contribute to merozoite egress. A new report also suggests that calcium-activated host proteases called calpains aid parasite exit, possibly by acting upon the host cytoskeleton. Together these discoveries reveal important new molecular players involved in the principal steps of egress by apicomplexans.

\section{Introduction}

Intracellular microorganisms are uniquely adapted to grow and develop within the confines of a host cell. This niche affords partial protection from host immune defences and provides the microbe a ready source of

Received 12 May, 2009; revised 29 June, 2009; accepted 6 July, 2009. ${ }^{*}$ For correspondence. E-mail vcarruth@umich.edu; Tel. (+1) 734763 2081; Fax (+1) 7347643562.

(C) 2009 Blackwell Publishing Ltd nutrients. The Apicomplexa are a group of obligate, intracellular protozoan parasites that include many species of medical and veterinary significance. During acute infection, these parasites use strategies that minimize the vulnerable time spent outside of the cell, in some cases abandoning an expired host cell for a fresh one in only a few seconds. Tools used to successfully make this transition include actin-myosin based gliding motility and specialized apical secretory organelles, which have been characterized largely from their role in host cell invasion. Gliding motility drives active cell invasion, which is assisted by secretory proteins that promote host cell adhesion and formation of a parasitophorous vacuole (PV) wherein parasite replication ensues.

Egress by intracellular pathogens is much less well understood than cell invasion. Parasite escape from the host cell requires the breaching of multiple barriers including the parasitophorous vacuole membrane (PVM), host cytosolic organelles and other membrane bound structures, the host cytoskeleton, and the host plasma membrane (HPM). Ideally, the parasite times the disruption of these barriers with completion of its replicative cycle to emerge from one infected cell fully prepared to infect the neighbouring host cell. Several recent studies support egress as an active phenomenon and have identified parasite proteins required for egress including the contribution of a pore-forming protein (PFP) for Toxoplasma gondii exit and a key role for parasite proteases in Plasmodium escape. A role for host calpain proteases has also been established recently, highlighting a novel host contribution to parasite egress. Here we discuss these and other recent developments and compare the emerging views of cell egress by apicomplexan parasites, with emphasis on the most intensively studied pathogens of this phylum, Plasmodium and Toxoplasma.

Acute infection: cycles of invasion, replication and Iytic egress

A hallmark of acute infection by Plasmodium and Toxoplasma is the repeated cycle of cell invasion, intracellular replication and parasite egress (Black and Boothroyd, 
2000). Cell invasion occurs by similar mechanisms in both parasites and begins with attachment of the zoite (merozoite or tachyzoite) to the host cell surface and orientation of the apical end of the parasite towards the HPM (Cowman and Crabb, 2006; Carruthers and Boothroyd, 2007). Secretion of proteins from the apical microneme and rhoptry organelles leads to formation of a moving junction, a structure composed of parasite proteins and host components that span the host cell membrane while maintaining contact with the parasite plasma membrane. The parasite uses its actin-myosin motor system to slide through the moving junction, thereby invaginating the HPM and encapsulating itself within the PV in the host cytosol. While the PV originates from the HPM, it is extensively modified by the parasite during asexual replication (Martin et al., 2007; Sinai, 2008). Finally, during egress the parasite secretes proteins into the PV, becomes motile and actively exits the cell in search of a new target cell.

\section{Signalling during egress: should I stay or should I go?}

Video microscopy observations of parasite egress indicate that it is a rapid, and therefore tightly regulated, event (Moudy et al., 2001; Glushakova et al., 2005). It is beneficial to the parasite to control the timing of departure, especially for Plasmodium where premature egress would be disastrous because the intermediate replicative stages are not invasive. Toxoplasma has somewhat greater flexibility in the timing of egress because daughter parasites are continually produced during intracellular replication. Studies in Toxoplasma have established a role for $\mathrm{K}^{+}$and $\mathrm{Ca}^{+2}$ ion fluxes in parasite motility and egress (reviewed in Lavine and Arrizabalaga, 2007). The parasite senses $\mathrm{K}^{+}$levels in its surroundings, maintaining a non-motile state while bathed in the high $\mathrm{K}^{+}$intracellular environment, and activating its motility system upon experiencing a drop in external $\mathrm{K}^{+}$(Moudy et al., 2001). Plasmodium sporozoites respond to $\mathrm{K}^{+}$fluxes in a similar manner (Kumar et al., 2007). Precisely how the parasite senses $\mathrm{K}^{+}$is not known; however, phospholipase $\mathrm{C}$, parasite intracellular calcium, and several $\mathrm{Ca}^{+2}$ responsive proteins play a role (Table 1), suggesting regulation by $\mathrm{Ca}^{+2}$-based signalling (Moudy et al., 2001). Indeed, several studies have shown that parasite intracellular $\mathrm{Ca}^{+2}$, released from intracellular stores via several distinct signalling pathways (Nagamune et al., 2008a), regulates motility by activating both the glideosome motility system and apical secretion of micronemal transmembrane adhesins, which engage the motor and act as a drive train to transduce power into motion (Lavine and Arrizabalaga, 2007). Potassium sensing ensures the motility system is off during intracellular replication but can be rapidly initiated for a hasty exit. Whether $\mathrm{K}^{+}$flux is a cue for natural egress at the end of the replicative cycle is not known. Metabolic or mechanical strain in parasite-laden infected host cells could cause $\mathrm{K}^{+}$to flood out of the cell, thereby activating exit. Interestingly, parasites have also been observed to exit host cells in the absence of parasite motility or potassium flux (Lavine and Arrizabalaga, 2008). Here, the authors observed that osmotic stress and host cell membrane tension had an effect on egress. Thus, perhaps the parasite uses $\mathrm{K}^{+}$sensing mainly for emergency purposes when, for example, under immune attack. In fact, Persson et al. (2008) have recently shown that Toxoplasma rapidly exits from cells undergoing apoptosis due to natural killer (NK) cell engagement of Fas ligand or delivery of perforin and granzymes (Fig. 1A). Rather than perishing in the dying cell the parasite remarkably reverses its fate by infecting the NK cell that instigated the attack (Persson et al., 2009). This establishes that the parasite can respond to extrinsic cues to exit a damaged or dying cell and therefore avoid going down with the ship.

Under normal circumstances, however, it is more likely that the parasite uses intrinsic cues to orchestrate its escape. One clue to how this might happen came with the recent identification of the egress signalling molecule called abscisic acid (ABA) (Nagamune et al., 2008b). In plants, ABA acts as a hormone that mediates growth and responses to environmental cues through cyclic ADPribose (cADPR) and calcium fluxes. In Toxoplasma, ABA induces the production of cADPR, which activates $\mathrm{Ca}^{+2}$ release from an internal membrane-bound pool (probably the endoplasmic reticulum), and this in turn triggers the secretion of microneme proteins and presumably also starts the parasite's motor (Fig. 1A, step 1). Although the parasite genome has candidate genes for ABA synthesis, their precise role remains to be determined. Reflecting its plant heritage, ABA is most likely synthesized in the apicoplast, an organelle derived from an algal endosymbiont. Interestingly, ABA levels remain low during parasite replication, but rapidly spike just prior to parasite egress. Thus, steep production of $A B A$ at the end of the replicative cycle might serve as an intrinsic cue for egress. The herbicide fluridone inhibited ABA synthesis and blocked parasite egress, but not growth or invasion. Furthermore, mice treated with fluridone survived inoculation with a lethal dose of Toxoplasma. Indeed, fluridone has also been shown to be effective against malaria, indicating common ABA pathways in apicomplexans. However, blocking ABA signalling in Toxoplasma tissue culture samples not only blocked egress, but also led to bradyzoite development. This suggests the absence of normal egress signals in the lytic cycle results in tachyzoite to bradyzoite differentiation and may regulate switching between these developmental stages. 
Table 1. Egress inducers, inhibitors and proteins.

\begin{tabular}{|c|c|c|c|c|}
\hline Egress inducers & Species & \multicolumn{2}{|c|}{ Mode of action } & References \\
\hline A23187a & T. gondii & \multicolumn{2}{|c|}{ Calcium ionophore } & Black et al. (2000) \\
\hline Dithiothreitol $^{a}$ & T. gondii & \multicolumn{2}{|c|}{ Nucleotide triphosphatase activation? } & Stommel et al. (1997) \\
\hline Nigericin $^{a}$ & T. gondii & \multicolumn{2}{|c|}{ Potassium ionophore } & Fruth and Arrizabalaga (2007) \\
\hline Ethanol $^{\mathrm{a}}$ & T. gondii & \multicolumn{2}{|c|}{ Calcium flux, possibly by activation of phospholipase C } & Carruthers et al. (1999) \\
\hline Streptolysin $\mathrm{O}^{\mathrm{a}}$ & T. gondii & \multirow{2}{*}{\multicolumn{2}{|c|}{$\begin{array}{l}\text { Permeabilization of HPM } \\
\text { Permeabilization of HPM }\end{array}$}} & Moudy et al. (2001) \\
\hline S. aureus $\alpha$-toxin ${ }^{a}$ & T. gondii & & Permeabilization of HPM & Moudy et al. (2001) \\
\hline Abscisic acid ${ }^{b}$ & T. gondii & \multicolumn{2}{|c|}{ Calcium flux via production of cADPR } & Nagamune et al. (2008b) \\
\hline NK cell ${ }^{b}$ & T. gondii & \multicolumn{2}{|c|}{ Induction of apoptosis } & Persson et al. (2009) \\
\hline Egress inhibitors $^{c}$ & Species & & Target & References \\
\hline U73122 & T. gondii & & TgPLC & Moudy et al. (2001) \\
\hline Cytochalasin $\mathrm{D}^{\mathrm{d}}$ & T. gondii & & Actin & $\begin{array}{l}\text { Schwartzman and Pfefferkorn (1983) } \\
\text { Black et al. (2000) } \\
\text { Lavine and Arrizabalaga (2007) }\end{array}$ \\
\hline Wortmannin & T. gondii & & Phosphoinositide 3-kinase & Caldas et al. (2007) \\
\hline Staurosporine & T. gondii & & Unknown serine/threonine kinase(s) & Caldas et al. (2007) \\
\hline Genistein & T. gondii & & Unknown tyrosine kinase(s) & Caldas et al. (2007) \\
\hline KT5926 & T. gondii & & TgCDPK1 & $\begin{array}{l}\text { Kieschnick et al. (2001) } \\
\text { Moudy et al. (2001) }\end{array}$ \\
\hline DCG104 & T. gondii and P. falcipa & arum & Host calpain1/2 & Chandramohanadas et al. (2009) \\
\hline Dimethylamiloride & T. gondii & & TgNHE1 & Arrizabalaga et al. (2004) \\
\hline MRT12113 & P. falciparum & & PfSUB1 & Yeoh et al. (2007) \\
\hline JCP104 & P. falciparum & & PfSUB1 & Arastu-Kapur et al. (2008) \\
\hline SAK1 & P. falciparum & & PfDPAP3 & Arastu-Kapur et al. (2008) \\
\hline Egress proteins & Species & & Function & References \\
\hline TgPLP1 & T. gondii & & Permeabilization of PVM (and HPM?) & Kafsack et al. (2009) \\
\hline Host Calpain $1 / 2$ & T. gondii and $P$. falciparun & & Cytoskeletal remodelling? & Chandramohanadas et al. (2009) \\
\hline PbECP1/PbSERA5 & P. berghei & & Sporozoite egress from oocyst & Aly et al. (2008) \\
\hline PfSUB1 & P. falciparum & & SERA maturation within the PV & $\begin{array}{l}\text { Yeoh et al. (2007); Arastu-Kapur et al. } \\
\text { (2008) }\end{array}$ \\
\hline PfDPAP3 & P. falciparum & & PfSUB1 maturation within the parasite & Arastu-Kapur et al. (2008) \\
\hline PbSIAP1 & P. berghei & & $\begin{array}{l}\text { Role in gliding locomotion, knockout has partial } \\
\text { oocyst egress defect }\end{array}$ & Engelmann et al. (2009) \\
\hline TgMyoA & T. gondii & & Gliding motility motor & Meissner et al. (2001) \\
\hline TgPRF & T. gondii & & Actin polymerization & Plattner et al. (2008) \\
\hline TgNHE1 & T. gondii & & Calcium homeostasis & Arrizabalaga et al. (2004) \\
\hline TgCDPK1 & T. gondii & & Induction of microneme secretion and motility & Moudy et al. (2001) \\
\hline PbMDV1/PEG3 & P. berghei & & Gamete egress from erythrocytes & Ponzi et al. (2009) \\
\hline
\end{tabular}

a. Artificial egress inducer.

b. Natural egress inducer.

c. Artificial egress inhibitors.

d. Blocks artificially induced egress and delays natural egress.

TgPLC, Toxoplasma gondii phospholipase C; TgCDPK1, Toxoplasma gondii calcium-dependent protein kinase 1; TgNHE1, Toxoplasma gondii sodium/hydrogen exchanger 1; PbECP1, Plasmodium berghei egress cysteine protease 1; PbSIAP1, Plasmodium berghei sporozoite invasionassociated protein 1; TgMyoA, Toxoplasma gondii myosin A, defective in induced egress; TgPRF, Toxoplasma gondii profilin.

\section{Poring out of the host cell}

Organisms throughout the tree of life use PFPs to breach membranes, usually those of another cell. The recent discovery that membrane attack complex/perforin (MACPF) proteins have structural homology to bacterial cholesterol-dependent cytolysins (Hadders et al., 2007; Rosado et al., 2007; 2008) provides a wealth of mechanistic insight into MACPF pore formation, and by extension, how apicomplexan parasites might use PLPs during infection. A brief overview of PFP function is given here and we refer the reader to the recent in-depth reviews for more information (Tweten, 2005; Lukoyanova and Saibil,
2008; Rosado et al., 2008). Most PFPs are secreted as monomers that bind to receptors on the target membrane, oligomerize, and insert to form a pore. PFPs are used for both host defence and microbial aggression. For example, pores created by the membrane attack complex (i.e. terminal components of the complement cascade) and perforin, aid in host immunity to microbial infections and malignancy. Bacteria also use PFPs on host membranes to escape from a vacuole after entry [e.g. Listeria (Dramsi and Cossart, 2002)] or to deliver bacterial factors into the host cell (e.g. Shigella and Yersinia; Blocker et al., 1999; Mota, 2006). With the exception of Cryptosporidium, potential PFPs have been identified in all apicom- 

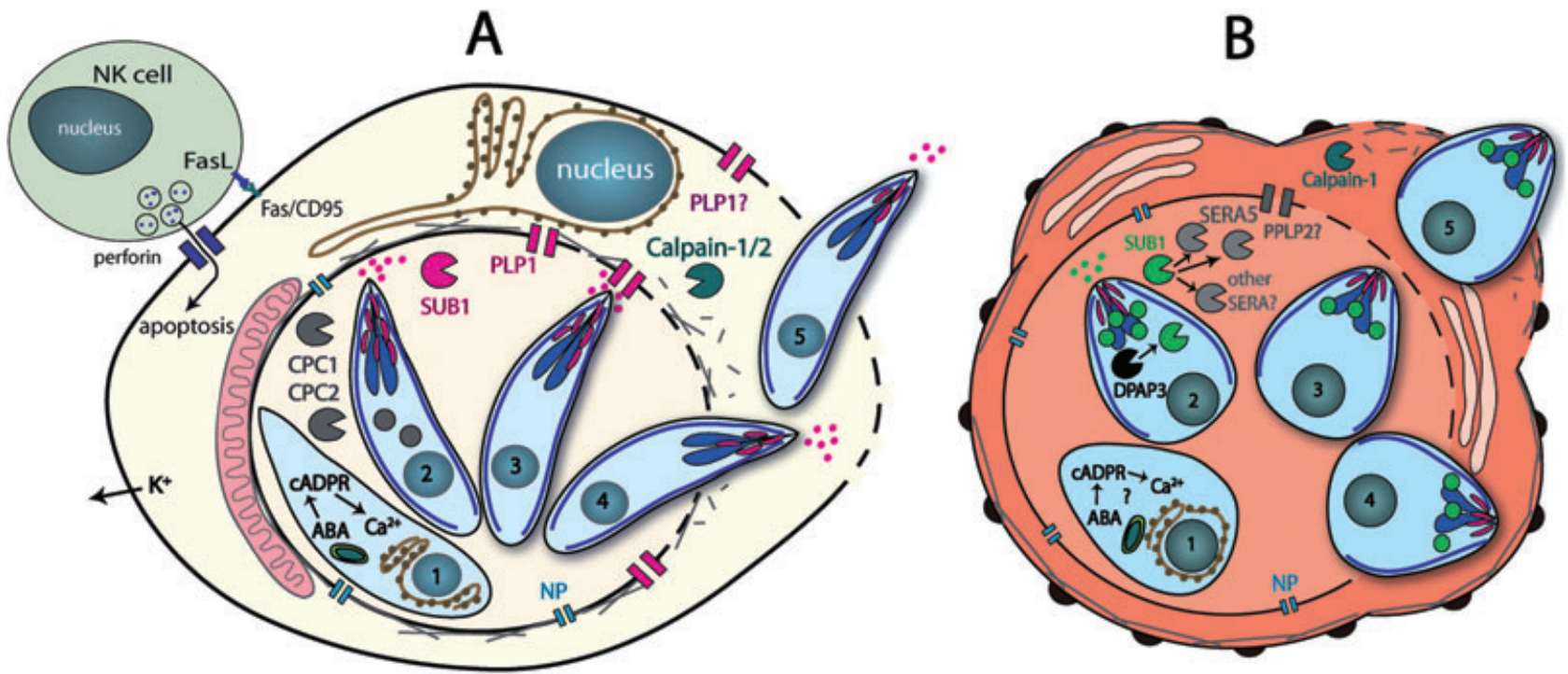

Fig. 1. Common steps in Toxoplasma tachyzoite and Plasmodium merozoite egress. Each step is indicated in the nucleus of Toxoplasma (A) or Plasmodium (B). (1) Transmission of an egress signal. (2) Protein secretion from micronemes (Toxoplasma) or exonemes (Plasmodium) and activation of the motility system (blue line underneath the zoite plasma membrane). (3) Disruption of the PVM. (4) Disruption of the host cytoskeleton and HPM. (5) Active exit from the host cell.

plexan genomes by sequence homology to the MACPF domain (Kafsack et al., 2009). The Plasmodium genome has five different MACPF-domain containing proteins, termed PPLP1-5 (Plasmodium perforin-like protein), and the Toxoplasma genome has two, TgPLP1 and TgPLP2 (Toxoplasma gondii perforin-like protein).

Permeabilization of the HPM and PVM a few minutes prior to Toxoplasma egress has been previously reported (Black et al., 2000; Moudy et al., 2001), and this membrane permeabilization was found to be calcium and parasite-dependent. However, the mechanism of membrane permeabilization remained unknown. Recently, TgPLP1 was found to be crucial for rapid parasite egress (Kafsack et al., 2009). Initial observations of TgPLP1deficient parasite cultures revealed the presence of parasites trapped within large spherical structures that were not seen in cultures of wild-type (WT) parasites or mutant parasites with restored expression of TgPLP1. Further examination of these structures showed parasites enveloped by one or two membranes, i.e. the PVM and/or HPM. Upon induced egress with a calcium ionophore, TgPLP1-deficient parasites activated motility and secreted microneme proteins but were significantly delayed in their time to host cell exit. Video microscopy of knockout parasites upon induced egress showed parasites became motile in the PV and actively attempted to cross the PVM, but with limited success. Eventual escape appears to be principally due to motility-based mechanical rupture of the PVM because the parasite's vigorous movements and probing of the PVM with its extruded conoid immediately preceded eventual egress. Moreover,
TgPLP1 expression was shown to be necessary for PVM permeabilization because a fluorescent protein expressed in the parasite PV failed to escape the PV upon induction of egress in the knockout, in contrast to WT or genetically complemented parasites where fluorescence was observed throughout the host cytosol. Whether TgPLP1 itself forms a pore has not been established, but it has all of the key structural features used for pore formation by MACPF/CDC proteins including two $\alpha$-helical elements that convert to amphipathic $\beta$-sheets to form a large oligomeric $\beta$-barrel pore. Co-infection, video microscopy and immunofluorescence experiments revealed WT parasites can donate TgPLP1 to aid in escape of knockout parasites from the same cell. This indicates that TgPLP1 can function in trans and from either side of the PVM, suggesting that the receptor for TgPLP1 pore formation is present on both sides of the membrane. It was proposed that TgPLP1 promotes rapid parasite egress by sufficiently weakening the PVM to allow escape of motile parasites (Fig. 1A, step 2). Alternatively or additionally, TgPLP1 pores could provide a conduit for other egress effector proteins, such as PV resident or secreted proteases and lipases, to access the host cytosol and disrupt the cytoskeleton and plasma membrane, thereby compromising the integrity of these other barriers to exit. TgPLP1-deficient parasites are highly attenuated ( 5-log decrease in $\left.L D_{100}\right)$ in the mouse model of toxoplasmosis, highlighting the importance of rapid egress in the normal course of infection and disease.

Whether TgPLP1 is responsible for permeabilization of the HPM observed 2-3 min prior to normal (uninduced) 
egress remains to be determined (Moudy et al., 2001). A small discharge of microneme material including TgPLP1 could initiate egress by allowing this protein to access the HPM via its own pore in the PVM. In this model, the ensuing release of $\mathrm{K}^{+}$from the cell would serve as a positive feedback loop to ignite the motility system and induce additional microneme secretion including TgPLP1 for destabilization of the PVM. Investigating the sequence of events associated with uninduced egress by TgPLP1deficient parasites should provide insight into the potential role of TgPLP1 in priming normal egress.

Although the three Plasmodium PPLPS (PPLP1, PPLP3 and PPLP5) characterized thus far function in cell traversal during tissue migration (Kadota et al., 2004; Ishino et al., 2005; Ecker et al., 2007) and do not play a role in parasite egress, cell traversal and egress both involve disruption of membrane barriers. Hence, the emerging theme is that apicomplexan parasites use PLPs as membrane-disrupting agents, but for distinct events in different developmental stages. The functions of the two other PPLPs have not been determined. Nonetheless, it is worth considering that they might function in cell egress. For example, PPLP2 is expressed in the Plasmodium intraerythrocytic stages, which do not traverse cells, but instead rely heavily on efficient egress to complete their lytic cycle. Also, PPLP5 is expressed in gametocytes, which also must egress from erythrocytes prior to mating. If, as first proposed by Ecker et al. (2007), PPLP2 and PPLP5 are secreted by merozoites and gametocytes, respectively, during egress, they could serve in a similar capacity to TgPLP1 to facilitate parasite escape from the $\mathrm{PV}$ and possibly also from the erythrocyte. Interestingly, the PLP family has undergone a large expansion to seven members in Theileria (Kafsack et al., 2009), probably reflecting multiple roles that may include vacuolar escape into the cytoplasm after entry and cell egress after replication.

One or more PPLPs may also function in egress of liver stage merozoites, which exit the liver within merozoitefilled vesicles called merosomes (Sturm et al., 2006; Baer et al., 2007). Merosomes, containing dozens to hundreds of merozoites, extrude from infected hepatocytes and pinch off into the blood circulation. These structures are thought to be escape pods that allow the parasite to exit the liver without being engulfed by macrophages or otherwise triggering an immune response. Merosomes appear to develop as a result of unconventional apoptosis of the infected hepatocyte when merozoites escape from the PV but do not breach the hepatocyte PM. Most merosomes remain intact in the host circulation until they reach the pulmonary microvasculature, where they disintegrate and release infectious merozoites (Baer et al., 2007). Releasing merozoites in lung capillaries assures close contact with erythrocytes and promotes rapid invasion to initiate the intraerythrocytic cycle. Thus, pore-formation could facilitate two steps along the journey from the liver to the erythrocyte: disruption of PVM upon merozoite maturation to promote PVM escape and merosome formation, and perturbation of the merosome membrane in the lung to liberate merozoites. Indeed, the PVM becomes permeable to large dyes and proteins prior to its breakdown during merozoite formation (Sturm et al., 2009). Although PPLPs have not been shown to play a role in egress from merosomes, this possibility deserves investigation.

\section{Proteolytic egress}

While we can only speculate on a role for PFPs in egress by Apicomplexa other than Toxoplasma, several recent studies have provided compelling evidence for proteases in Plasmodium egress (see also Blackman, 2008 for a recent in depth review). Protease inhibitors have proved invaluable tools in analysing Plasmodium biology and have shown that egress is a protease-dependent function. While multiple models for merozoite egress have been proposed, evidence is accumulating in support of a model in which the PVM is disrupted prior to the HPM of the erythrocyte (Fig. 1B). For example, using GFP transgenic parasites with GFP targeted to either the PV or host cytosol, Wickham and colleagues showed that the PV GFP signal expanded beyond the PV late in schizogony, indicating PVM disruption (Wickham et al., 2003). This was confirmed by immuno-electron microscopy with antibodies to S-antigen, which is normally confined to the PV but is found throughout the erythrocyte cytoplasm late in schizogony. Further evidence for a model where the parasite egresses in a stepwise manner came from observations that disruption of each limiting membrane is sensitive to different protease inhibitors (Salmon et al., 2001; Wickham et al., 2003; Soni et al., 2005). The cysteine and serine protease inhibitors leupeptin and antipain have been observed to block HPM disruption. While previous reports concluded the cysteine protease inhibitor E-64 blocked PVM disruption, Glushakova et al. recently observed a block in erythrocyte membrane disruption upon E-64 treatment (Glushakova et al., 2009). However, the targets of these inhibitors have yet to be determined. Plasmodium expresses other proteases involved in haemoglobin digestion (plasmepsins, falcipain-2) and these also have activity against host cytoskeletal proteins (Le Bonniec et al., 1999). However, these proteases are not required for blood stage growth and knockout parasites are not defective in egress (Omara-Opyene et al., 2004; Sijwali and Rosenthal, 2004; Sijwali et al., 2006), which indicates redundancy in protease function and that the haemoglobinases may not be not essential for egress.

Merozoite egress from erythrocytes appears to involve a cascade of proteolytic activation and activity. DPAP3, a 
cathepsin $\mathrm{C}$-like cysteine protease, is required for maturation of PfSUB1, a subtilisin-like protease (Fig. 1B, step 2). DPAP3 also functions as an AMA1 maturase and may be involved in the processing of other secreted proteins (Arastu-Kapur et al., 2008). PfSUB1 is an essential protein expressed in late blood stage parasites and is secreted from merozoite exonemes into the PV prior to egress. Exonemes are novel organelles seen in merozoites during late schizogony (Yeoh et al., 2007). Once in the PV, PfSUB1 processes serine-rich antigen 5 (SERA5) and potentially other SERA proteins (Yeoh et al., 2007; Arastu-Kapur et al., 2008), as well as merozoite surface proteins (Koussis et al., 2009).

The serine-repeat antigen (SERA) proteins are PV proteins with a putative papain-like protease domain. Although evidence of proteolytic activity has yet to be demonstrated, it is possible that PfSUB1 processing of SERA5 and other SERAs results in their activation and proteolysis of host cytoskeletal proteins to aid in parasite egress (Yeoh et al., 2007) (Fig. 1B, step 2). Alternatively, SERAs might activate other parasite or host proteins that promote parasite egress. While SERA substrates are not known, several of the SERAs appear to be essential at different stages of the life cycle. PfSERA3, 4, 5, 6 and 9 are found in the PV of blood stage $P$. falciparum and are highly abundant during the schizont stage prior to egress. Among these, PfSERA5 and PfSERA6 are essential proteins. PfSERA5 processing from a precursor to mature form is linked to egress because this putative activation step occurs coincident with parasite exit from the erythrocyte. PfSERA5 inhibition by a cyclical peptide halts late stage development of intraerythrocytic parasites (Fairlie et al., 2008), possibly by blocking egress. Also, a Plasmodium berghei SERA orthologue, ecp1/PbSERA5, is required for sporozoite egress from oocysts. Knockout sporozoites remained trapped inside the oocyst and had altered motility compared with WT parasites (Aly et al., 2008). Furthermore, WTs and knockout parasites had differences in an inner oocyst wall component, circumsporozoite protein (CSP) processing, and WT samples in the presence of E-64 displayed the same CSP processing as the KO samples with or without E-64 treatment. This suggests PbSERA5 is involved in CSP proteolytic maturation and demonstrates that oocyst exit is an active process mediated by the parasite. SERA proteins may also have a role in merozoite egress from the hepatocyte. PbSERA3 is expressed late in the liver stage, proteolytically processed, and translocated to the host cytosol upon PVM rupture. However, under E-64 treatment, PbSERA3 remains associated with the parasite and the PVM remains intact. PbSERA3 is thought to play a role in host cell death by activating other parasite or host proteins (Schmidt-Christensen et al., 2008).
Although Toxoplasma has no SERA orthologues, other proteases may have a role in egress. Two cathepsin C-like proteases are secreted into the PV via dense granules during replication (Que et al., 2007). Involvement in nutrient acquisition has been suggested for these TgCPCs due to their exopeptidase activity, but a role in egress has not been excluded. Toxoplasma also secretes at least two additional proteases including a subtilisin called TgSUB1 and a metalloprotease (Zhou et al., 2005) termed Toxolysin 4 (TLN4, J. Laliberté and V. Carruthers, unpublished). However, TgSUB1-deficient parasites are viable, indicating this protease is not essential for the lytic cycle (K. Kim, pers. comm.). The function of the TLN4 remains to be determined. Clearly, more work is necessary to determine if proteases contribute to Toxoplasma egress.

A host calcium-dependent protease, calpain-1, is also required for efficient parasite egress of Plasmodium and Toxoplasma (Fig. 1, steps 3-4) (Chandramohanadas et al., 2009). Treatment of $P$. falciparum-infected erythrocytes with an irreversible cysteine protease inhibitor, DCG04, did not affect parasite growth but prevented parasite release from the host cell. Selective extraction of treated cells identified host calpain-1 as the target of the inhibitor. Calpain-1 was observed in the cytoplasm of the infected host cell until the schizont stage of parasite growth, when it shifted to the membrane, indicating calcium binding and activation. Calpain-1 extraction from erythrocytes prevented parasite egress and led to growth arrest in the schizont stage, whereas reconstitution with recombinant calpain-1 restored normal growth development. Host calpain was also found to be required for Toxoplasma gondii egress from human host cells treated with an siRNA that targeted both calpain-1 and 2. Infection of calpain-deficient cells led to the appearance of spherical structures, similar to those seen in TgPLP1-deficient parasites, containing tachyzoites that were unable to exit host cells. While P. falciparum merozoites were never detected egressing erythrocytes lacking calpain-1, some T. gondii parasites were able exit from calpain-deficient fibroblasts, indicating redundancy or non-essentiality in calpain's role in Toxoplasma egress. As calpains are well known for their role in cytoskeletal remodelling (Goll et al., 2003), it is reasonable to think that they aid egress by helping to dismantle the host cytoskeleton.

\section{Common steps in egress and outstanding questions}

It is worth considering the emerging similarities of egress by apicomplexans as a conceptual framework for future work. Although some distinctions due to the specific situations of different apicomplexans are likely to exist, the basic principles of parasite egress appear to be similar and can be grouped into five steps (Fig. 1). These steps 
may partially overlap and may not occur in the same sequence for all parasites. (i) The parasite produces an intrinsic egress cue, such as ABA, or senses an extrinsic signal to egress, such as immune attack or a drop in host $\mathrm{K}^{+}$. (ii) The parasite activates motility and secretes egress effector proteins. (iii) The PVM is disrupted through the concerted action of PFPs, proteases, and perhaps other effector proteins. (iv) Parasite and host proteins act to disrupt the host cytoskeleton and HPM. (v) The parasite uses its motility system to escape from the decaying host cell in search of a new host cell to infect.

While recent studies have shed new light on the molecular mechanisms of cell egress by apicomplexan parasites, many outstanding questions remain to be addressed. Do Plasmodium proteases have direct or indirect roles in egress for activating other effector proteins or host cytoskeleton digestion? For direct roles, how do the proteases in the PV gain access to the host cytosol: via the actions of PFPs or secreted lipases? How do the parasites prevent self-damage from PFPs? Do secretory proteases contribute to Toxoplasma egress? Are the signals mediating Plasmodium egress similar to those of Toxoplasma? Do secretory proteins from other organelles (e.g. rhoptries or dense granules) contribute to egress? Interfering with egress has been shown to be an effective method of disrupting parasite transmission and pathogenesis. Thus, the study of host cell exit in apicomplexans is expected to reveal conserved and unique roles for parasite and host proteins, thereby revealing potential new targets of therapeutic intervention.

\section{Acknowledgements}

We thank all of the investigators who have contributed to the understanding of apicomplexan egress, and we apologize to those whose work we were unable to describe due to space limitations. We also gratefully acknowledge Björn Kafsack and My-Hang Huynh for helpful discussions and for critically reading this manuscript prior to submission. Egress related studies in our laboratory are supported by Grant RO1Al046675 from the National Institutes of Health.

\section{References}

Aly, A.S., Mikolajczak, S.A., Rivera, H.S., Camargo, N., Jacobs-Lorena, V., Labaied, M., et al. (2008) Targeted deletion of SAP1 abolishes the expression of infectivity factors necessary for successful malaria parasite liver infection. Mol Microbiol 69: 152-163.

Arastu-Kapur, S., Ponder, E.L., Fonovic, U.P., Yeoh, S., Yuan, F., Fonovic, M., et al. (2008) Identification of proteases that regulate erythrocyte rupture by the malaria parasite Plasmodium falciparum. Nat Chem Biol 4: 203213.

Arrizabalaga, G., Ruiz, F., Moreno, S., and Boothroyd, J.C. (2004) Ionophore-resistant mutant of Toxoplasma gondii reveals involvement of a sodium/hydrogen exchanger in calcium regulation. J Cell Biol 5: 653-662.

Baer, K., Klotz, C., Kappe, S.H., Schnieder, T., and Frevert, U. (2007) Release of hepatic Plasmodium yoelii merozoites into the pulmonary microvasculature. PLoS Pathog 3: e171.

Black, M.W., and Boothroyd, J.C. (2000) Lytic cycle of Toxoplasma gondii. Microbiol Mol Biol Rev 64: 607-623.

Black, M.W., Arrizabalaga, G., and Boothroyd, J.C. (2000) lonophore-resistant mutants of Toxoplasma gondii reveal host cell permeabilization as an early event in egress. Mol Cell Biol 20: 9399-9408.

Blackman, M.J. (2008) Malarial proteases and host cell egress: an 'emerging' cascade. Cell Microbiol 10: 19251934.

Blocker, A., Gounon, P., Larquet, E., Niebuhr, K., Cabiaux, V., Parsot, C., and Sansonetti, P. (1999) The tripartite type III secreton of Shigella flexneri inserts IpaB and IpaC into host membranes. J Cell Biol 147: 683-693.

Caldas, L.A., de Souza, W., and Attias, M. (2007) Calcium ionophore-induced egress of Toxoplasma gondii shortly after host cell invasion. Vet Parasitol 3-4: 210-220.

Carruthers, V., and Boothroyd, J.C. (2007) Pulling together: an integrated model of Toxoplasma cell invasion. Curr Opin Microbiol 10: 83-89.

Carruthers, V., Moreno, S.N.J., and Sibley, L.D. (1999) Ethanol and acetaldehyde elevate intracellular calcium and stimulate microneme discharge in Toxoplasma gondii. Biochem J 2: 379-386.

Chandramohanadas, R., Davis, P.H., Beiting, D.P., Harbut, M.B., Darling, C., Velmourougane, G., et al. (2009) Apicomplexan parasites co-opt host calpains to facilitate their escape from infected cells. Science 5928: 794-797.

Cowman, A.F., and Crabb, B.S. (2006) Invasion of red blood cells by malaria parasites. Cell 124: 755-766.

Dramsi, S., and Cossart, P. (2002) Listeriolysin O: a genuine cytolysin optimized for an intracellular parasite. J Cell Biol 156: 943-946.

Ecker, A., Pinto, S.B., Baker, K.W., Kafatos, F.C., and Sinden, R.E. (2007) Plasmodium berghei: Plasmodium perforin-like protein 5 is required for mosquito midgut invasion in anopheles stephensi. Exp Parasitol 116: 504-508.

Engelmann, S., Silvie, O., and Matuschewski, K. (2009) Disruption of Plasmodium sporozoite transmission by depletion of sporozoite invasion-associated protein 1. Eukaryot Cell 4: 640-648.

Fairlie, W.D., Spurck, T.P., McCoubrie, J.E., Gilson, P.R., Miller, S.K., McFadden, G.I., et al. (2008) Inhibition of malaria parasite development by a cyclic peptide that targets the vital parasite protein SERA5. Infect Immun 76: 4332-4344.

Fruth, I.A., and Arrizabalaga, G. (2007) Toxoplasma gondii: induction of egress by the potassium ionophore nigericin. Int J Parasitol 14: 1559-1567.

Glushakova, S., Yin, D., Li, T., and Zimmerberg, J. (2005) Membrane transformation during malaria parasite release from human red blood cells. Curr Biol 15: 1645-1650.

Glushakova, S., Mazar, J., Hohmann-Marriott, M.F., Hama, E., and Zimmerberg, J. (2009) Irreversible effect of cysteine protease inhibitors on the release of malaria parasites from infected erythrocytes. Cell Microbiol 11: 95-105. 
Goll, D.E., Thompson, V.F., Li, H., Wei, W., and Cong, J. (2003) The calpain system. Physiol Rev 83: 731-801.

Hadders, M.A., Beringer, D.X., and Gros, P. (2007) Structure of C8alpha-MACPF reveals mechanism of membrane attack in complement immune defense. Science 317: 1552-1554.

Ishino, T., Chinzei, Y., and Yuda, M. (2005) A Plasmodium sporozoite protein with a membrane attack complex domain is required for breaching the liver sinusoidal cell layer prior to hepatocyte infection. Cell Microbiol 7: 199208.

Kadota, K., Ishino, T., Matsuyama, T., Chinzei, Y., and Yuda, M. (2004) Essential role of membrane-attack protein in malarial transmission to mosquito host. Proc Natl Acad Sci USA 101: 16310-16315.

Kafsack, B.F., Pena, J.D., Coppens, I., Ravindran, S., Boothroyd, J.C., and Carruthers, V.B. (2009) Rapid membrane disruption by a perforin-like protein facilitates parasite exit from host cells. Science 323: 530-533.

Kieschnick, H., Wakefield, T., Narducci, C.A., and Beckers, C. (2001) Toxoplasma gondii attachment to host cells is regulated by a calmodulin- like domain protein kinase. J Biol Chem 15: 12369-12377.

Koussis, K., Withers-Martinez, C., Yeoh, S., Child, M., Hackett, F., Knuepfer, E., et al. (2009) A multifunctional serine protease primes the malaria parasite for red blood cell invasion. EMBO J 28: 725-735.

Kumar, K.A., Garcia, C.R., Chandran, V.R., Van Rooijen, N., Zhou, Y., Winzeler, E., and Nussenzweig, V. (2007) Exposure of Plasmodium sporozoites to the intracellular concentration of potassium enhances infectivity and reduces cell passage activity. Mol Biochem Parasitol 156: 3240.

Lavine, M.D., and Arrizabalaga, G. (2007) Invasion and egress by the obligate intracellular parasite Toxoplasma gondii: potential targets for the development of new antiparasitic drugs. Curr Pharm Des 13: 641-651.

Lavine, M.D., and Arrizabalaga, G. (2008) Exit from host cells by the pathogenic parasite Toxoplasma gondii does not require motility. Eukaryot Cell 7: 131-140.

Le Bonniec, S., Deregnaucourt, C., Redeker, V., Banerjee, R., Grellier, P., Goldberg, D.E., and Schrevel, J. (1999) Plasmepsin II, an acidic hemoglobinase from the Plasmodium falciparum food vacuole, is active at neutral $\mathrm{pH}$ on the host erythrocyte membrane skeleton. J Biol Chem 274: 14218-14223.

Lukoyanova, N., and Saibil, H.R. (2008) Friend or foe: The same fold for attack and defense. Trends Immunol 29: 51-53.

Martin, A.M., Liu, T., Lynn, B.C., and Sinai, A.P. (2007) The Toxoplasma gondii parasitophorous vacuole membrane: transactions across the border. J Eukaryot Microbiol 54: 25-28.

Meissner, M., Brecht, S., Bujard, H., and Soldati, D. (2001) Modulation of myosin A expression by a newly established tetracycline repressor-based inducible system in Toxoplasma gondii. Nucleic Acids Res 22: E115, 1-10.

Mota, L.J. (2006) Type III secretion gets an LcrV tip. Trends Microbiol 14: 197-200.

Moudy, R., Manning, T.J., and Beckers, C.J. (2001) The loss of cytoplasmic potassium upon host cell breakdown triggers egress of Toxoplasma gondii. J Biol Chem 276: 41492-41501.

Nagamune, K., Moreno, S.N., Chini, E.N., and Sibley, L.D. (2008a) Calcium regulation and signaling in apicomplexan parasites. Subcell Biochem 47: 70-81.

Nagamune, K., Hicks, L.M., Fux, B., Brossier, F., Chini, E.N., and Sibley, L.D. (2008b) Abscisic acid controls calciumdependent egress and development in Toxoplasma gondii. Nature 451: 207-210.

Omara-Opyene, A.L., Moura, P.A., Sulsona, C.R., Bonilla, J.A., Yowell, C.A., Fujioka, H., et al. (2004) Genetic disruption of the Plasmodium falciparum digestive vacuole plasmepsins demonstrates their functional redundancy. J Biol Chem 279: 54088-54096.

Persson, C.M., Lambert, H., Vutova, P.P., DellacasaLindberg, I., Nederby, J., Yagita, H., et al. (2009) Transmission of Toxoplasma gondii from infected dendritic cells to natural killer cells. Infect Immun 77: 970-976.

Persson, K.E., McCallum, F.J., Reiling, L., Lister, N.A., Stubbs, J., Cowman, A.F., et al. (2008) Variation in use of erythrocyte invasion pathways by Plasmodium falciparum mediates evasion of human inhibitory antibodies. $J$ Clin Invest 118: 342-351.

Plattner, F., Yarovinsky, F., Romero, S., Didry, D., Carlier, M.F., Sher, A., and Soldati-Favre, D. (2008) Toxoplasma profilin is essential for host cell invasion and TLR11dependent induction of an interleukin-12 response. Cell Host Microbe 3: 77-87.

Ponzi, M., Siden-Kiamos, I., Bertuccini, L., Curra, C., Kroeze, H., Camarda, G., et al. (2009) Egress of Plasmodium berghei gametes from their host erythrocyte is mediated by the MDV-1/PEG3 protein. Cell Microbiol 11: 12721288.

Que, X., Engel, J.C., Ferguson, D., Wunderlich, A., Tomavo, S., and Reed, S.L. (2007) Cathepsin cs are key for the intracellular survival of the protozoan parasite, Toxoplasma gondii. J Biol Chem 282: 4994-5003.

Rosado, C.J., Buckle, A.M., Law, R.H., Butcher, R.E., Kan, W.T., Bird, C.H., et al. (2007) A common fold mediates vertebrate defense and bacterial attack. Science 317: 1548-1551.

Rosado, C.J., Kondos, S., Bull, T.E., Kuiper, M.J., Law, R.H., Buckle, A.M., et al. (2008) The MACPF/CDC family of pore-forming toxins. Cell Microbiol 9: 1765-1774.

Salmon, B.L., Oksman, A., and Goldberg, D.E. (2001) Malaria parasite exit from the host erythrocyte: a two-step process requiring extraerythrocytic proteolysis. Proc Natl Acad Sci USA 98: 271-276.

Schmidt-Christensen, A., Sturm, A., Horstmann, S., and Heussler, V.T. (2008) Expression and processing of Plasmodium berghei SERA3 during liver stages. Cell Microbiol 10: 1723-1734.

Schwartzman, J.D., and Pfefferkorn, E.R. (1983) Immunofluorescent localization of myosin at the anterior pole of the coccidian, Toxoplasma gondii. J Protozool 4: 657-661.

Sijwali, P.S., and Rosenthal, P.J. (2004) Gene disruption confirms a critical role for the cysteine protease falcipain-2 in hemoglobin hydrolysis by Plasmodium falciparum. Proc Natl Acad Sci USA 101: 4384-4389.

Sijwali, P.S., Koo, J., Singh, N., and Rosenthal, P.J. (2006) Gene disruptions demonstrate independent roles for the 
four falcipain cysteine proteases of Plasmodium falciparum. Mol Biochem Parasitol 150: 96-106.

Sinai, A.P. (2008) Biogenesis of and activities at the Toxoplasma gondii parasitophorous vacuole membrane. Subcell Biochem 47: 155-164.

Soni, S., Dhawan, S., Rosen, K.M., Chafel, M., Chishti, A.H., and Hanspal, M. (2005) Characterization of events preceding the release of malaria parasite from the host red blood cell. Blood Cells Mol Dis 35: 201-211.

Stommel, E.W., Ely, K.H., Schwartzman, J.D., and Kasper, L.H. (1997) Toxoplasma gondii: dithiol-induced Ca2+ flux causes egress of parasites from the parasitophorous vacuole. Exp Parasitol 2: 88-97.

Sturm, A., Amino, R., van de Sand, C., Regen, T., Retzlaff, S., Rennenberg, A., et al. (2006) Manipulation of host hepatocytes by the malaria parasite for delivery into liver sinusoids. Science 313: 1287-1290.

Sturm, A., Graewe, S., Franke-Fayard, B., Retzlaff, S., Bolte, S., Roppenser, B., et al. (2009) Alteration of the parasite plasma membrane and the parasitophorous vacuole membrane during exo-erythrocytic development of malaria parasites. Protist 160: 51-63.

Tweten, R.K. (2005) Cholesterol-dependent cytolysins, a family of versatile pore-forming toxins. Infect Immun 73: 6199-6209.

Wickham, M.E., Culvenor, J.G., and Cowman, A.F. (2003) Selective inhibition of a two-step egress of malaria parasites from the host erythrocyte. J Biol Chem 278: 3765837663.

Yeoh, S., O’Donnell, R.A., Koussis, K., Dluzewski, A.R., Ansell, K.H., Osborne, S.A., et al. (2007) Subcellular discharge of a serine protease mediates release of invasive malaria parasites from host erythrocytes. Cell 131: 10721083.

Zhou, X.W., Kafsack, B.F., Cole, R.N., Beckett, P., Shen, R.F., and Carruthers, V.B. (2005) The opportunistic pathogen Toxoplasma gondii deploys a diverse legion of invasion and survival proteins. J Biol Chem 280: 34233-34244. 